\title{
ssuons
}

IBERO-AMERICANOS

\section{Desafiando la bipolaridad: la independencia diplomática del gobierno democratacristiano en Chile y su acercamiento con el "mundo socialista" (1964-1970)}

\author{
Desafiando a bipolaridade: A independência diplomática do governo Cristão Democrata \\ e sua reaproximação com o "mundo socialista" \\ Defying bipolarity: the diplomatic independence of the Christian Democratic Government
in Chile and its rapprochement with the "Socialist World" (1964-1970)
}

Rafael Pedemonte*

\begin{abstract}
Resumen: Estos últimos años han sido testigos de una renovación historiográfica tendiente a subrayar el rol de los países latinoamericanos en el desarrollo de la Guerra Fría. Siguiendo esta línea, lejos de constituir un actor pasivo, el gobierno democratacristiano de Eduardo Frei Montalva en Chile (1964-1970) hizo prueba de una real independencia diplomática, desafiando la fidelidad estricta a los lineamientos de Estados Unidos y construyendo nuevos puentes con los países socialistas. Es así como la administración Frei, estimulada por el Ministro de Asuntos Exteriores Gabriel Valdés, optó desde temprano por establecer relaciones oficiales con la URSS, así como con otros cinco "Estados socialistas", propulsando de esta manera los vínculos políticos, económicos y culturales con el "mundo del Este". Desde 1968, esta voluntad internacional facilitó igualmente el acercamiento comercial con la China maoísta y con la Revolución Cubana, con la cual La Moneda firmó un acuerdo de intercambio agrario, poniendo fin a la política de aislamiento continental contra Cuba decretada en la OEA. La actitud de la diplomacia chilena generó una airada polémica en el contexto latinoamericano y suscitó el reconocimiento de Fidel Castro, quien con anterioridad había atacado violentamente a Eduardo Frei y a su proyecto de "Revolución en Libertad". Todos estos antecedentes nos permiten constatar que, en gran medida, la política exterior de Salvador Allende tenía sus raíces en la apertura del periodo anterior.
\end{abstract}

Palabras clave: Eduardo Frei; Cuba; América Latina; Guerra Fría; URSS.

Resumo: A última década testemunhou uma renovação historiográfica com o objetivo de enfatizar o papel dos países Latino Americanos durante a guerra fria. Seguindo esta tendência, este artigo argumenta que longe de ser um ator passivo, o governo Cristão Democrata de Eduardo Frei (1964-1970) conduziu uma política externa independente, desafiando a estrita lealdade aos interesses Americanos, construindo novas pontes com o campo socialista. Desde cedo, a administração de Frei, encorajada pelo Ministro de Relações Exteriores Gabriel Valdés, decidiu estabelecer relações diplomáticas com a URRS, e, imediatamente após isto, com outros cinco países do leste Europeu, iniciando conexões políticas, econômicas e culturais com o bloco soviético. Além disso, desde 1968, esta nova abordagem facilitou a aproximação comercial com a China de Mao, e a Revolução Cubana, com a qual Santiago assinou um acordo agrícola, encerrando a política de isolamento contra Cuba praticada pela OAS. A atitude da diplomacia chilena deu início à fortes controvérsias dentro do contexto Latino Americano e resultou no reconhecimento de Fidel Castro, que por sua vez havia atacado a "revolução em liberdade" de Eduardo Frei. Todos estes elementos contribuem para que possamos constatar que, em uma larga medida, a política externa de Salvador Allende, desde 1970, tem raízes na abertura internacional praticada anteriormente.

Palavras-chave: Eduardo Frei; Cuba; América Latina; Guerra Fria; URSS.

* Historiador y asistente de investigación en la Universidad de Gante, Bélgica.

dados biográficos_biographic data 


\begin{abstract}
The last decade has witnessed a historiographical renewal aiming to emphasize the role played by the Latin American countries during the Cold War. Following this tendency, the article argues that far from being a passive actor, Eduardo Frei's Chilean Christian Democratic Government (1964-1970) conducted an independent foreign policy, defying the strict loyalty to the Americanpriorities and building new bridges with the Socialist camp. Very soon, Frei's administration, encouraged by the Minister of Foreign Affairs Gabriel Valdés, decided to establish diplomatic relationships with the USSR, and, immediately after that, with five other Eastern countries, prompting political, economic and cultural links with the Soviet Bloc. Moreover, since 1968, this renewed international approach facilitated a commercial rapprochement with Mao's China and the Cuban Revolution, with which Santiago signed an agriculture agreement, marking the end of the isolation policy against Cuba contracted in OAS. The attitude of the Chilean diplomacy triggered a strident controversy in the Latin American context and resulted in Fidel Castro's recognition, a leader who had previously attacked Eduardo Frei's "Revolution in Liberty". All these elements allow us to state that, to a large extent, Salvador Allende's foreign policy since 1970 had its roots in the earlier global international opening.
\end{abstract}

Keywords: Eduardo Frei; Cuba; Latin America; Cold War; USSR.

\section{Introducción}

De las cobrizas laderas de Rancagua hasta La Habana, desde la patria del cobre a la tierra de la caña.

Digamos salud con vino, digamos ¡Salud Fidel!

Hoy nuestros pueblos se hermanan

y nuestros caldos estallan en las bodegas cubanas (Vino chileno para Cuba: Antología poética, 1972, p. 66-67).

La renovación historiográfica de la última década en torno a la Guerra Fría y a sus repercusiones transnacionales ha producido una miríada de contribuciones estimulantes, muchas de las cuales buscan reevaluar el peso en el entramado mundial de regiones tradicionalmente marginadas del análisis. Es así como una nueva generación de investigadores, provenientes de diversas instituciones del orbe, ha tendido a examinar de manera más sutil el ascendiente del continente latinoamericano en la tumultuosa geopolítica de la segunda mitad del siglo XX. Mediante estos esfuerzos, la imagen de una América Latina pasiva, receptora impotente de influencias ideológicas o víctima de estrategias hegemónicas, ha dado paso a una apreciación diferente, menos binaria. Estos resultados provechosos han puesto el acento en el carácter protagónico de "las Américas", así como en la capacidad de las naciones del subcontinente de definir y aplicar políticas independientes y no por ello menos determinantes (BRANDS, 2010; GONZÁLEZ y PALIERAKI, 2013; HARMER y RIQUELME, 2014; SPENCER y GILBERT, 2008).
No obstante, dichas constataciones suelen justificarse mayoritariamente a través del ejemplo - revelador, por supuesto-de la Revolución Cubana, un proyecto que bajo el liderazgo inconfundible de Fidel Castro, logró llevar a cabo una gestión internacional parcialmente autónoma. En efecto, especialmente en la década de 1960, la Isla del Caribe impuso auténticos desafíos a las potencias preponderantes, poniendo a prueba tanto la paciencia de la nación aliada - la Unión Soviética - como la hegemonía regional del "enemigo del Norte", los Estados Unidos. Pero las particularidades innegables del caso cubano no debieran ocultar otras experiencias significativas en el contexto latinoamericano. Pocos se han esmerado en destacar que el gobierno democratacristiano en Chile presidido por Eduardo Frei Montalva (1964-1970) se transformó también en un actor gravitante de la "Guerra Fría interamericana" (HARMER, 2011), gozando además de un amplio margen de independencia en sus definiciones internacionales. El proyecto reformistas de Frei y de sus colaboradores (la "Revolución en Libertad"), sustentado por un programa ambicioso de transformaciones sociales y económicas, sumado a una osada apertura diplomática, fue percibido por muchos observadores como una alternativa "progresista" al modelo más radical y autoritario emanado desde La Habana.

Incluso los miembros de la OTAN, poco propensos a dirigir sus miradas hacia los países de más allá del océano Atlántico, optaron por crear, en septiembre de 1961, un activo Grupo de Expertos sobre América Latina (Archivos OTAN, C-R(61)48, 5 oct. 1961). Reunidos en este nuevo organismo con el objeto de responder a la "penetración comunista" en territorio latinoamericano - irrefutable a partir de abril 1961, cuando Fidel Castro asumió abiertamente el carácter 
"socialista" de su Revolución-, los especialistas de la Alianza Atlántica identificaron en el gobierno del Partido Demócrata Cristiano (PDC) una "experiencia que merece el apoyo y el interés participativo de todos los miembros de la OTAN". La consolidación del gobierno de Frei era deseable, ya que "ofrecería una alternativa respecto a las tentaciones del Castrocomunismo" (Archivos OTAN, C-M(65)26, 23 abr. 1965, p. 4). Algo similar fue percibido por la Casa Blanca, cuyos consejeros vieron en el PDC"la mejor esperanza para la democracia popular en América Latina" (BRANDS, 2010, p. 106). Mediante el apoyo masivo que Washington otorgó a la candidatura de Frei, los dirigentes norteamericanos se proponían popularizar un nuevo y audaz proyecto de cambio; una "revolución" no marxista ejercida dentro de los márgenes de la democracia y que, en caso de resultar exitosa, podría ser susceptible de debilitar el influjo de los cubanos (FERMANDOIS, 2004, p. 297-302).

Pero no solo el mundo occidental vio con buenos ojos el triunfo de Frei. Moscú tomó rápidamente conciencia de las ventajas ofrecidas por la nueva administración, un gobierno que, sin exigir ayuda a los países del Este, brindaba sin embargo una plataforma adecuada para ampliar las influencias soviéticas en un territorio históricamente dominado por la superpotencia rival. El latinoamericanista Iósif Grigulevich -más tarde conocido como uno de los más temibles espías de la Guerra Fría- destacaba ya en 1965 que Frei "estaba cumpliendo sus promesas", abriendo una "nueva página de la historia de Chile" mediante una "revolución pacifica" yuna "firme voluntad reformista" (GRIGULEVICH, 1970, p. 129). Incluso los dirigentes de la Cuba revolucionaria -que aprehensivos frente al poder de radiación de la "Revolución en Libertad" no dudaron en un comienzo en atacarairadamenteal Presidente chileno- hallaron hacia fines de los años 1960 más de una razón para estar satisfechos con la política exterior de Frei y, en particular, de su Ministro de Asuntos Exteriores Gabriel Valdés.

Esta relativa armonía con el "mundo socialista" tiene sus raíces en el programa que La Moneda intentó impulsar desde fines de 1964 y que conllevó a una reformulación de la posición de Chile en el mundo. Más allá de la reanudación de relaciones oficiales con Moscú, y con cinco otros países de la esfera del Este, el gobierno de Frei acentuó sus retos a la "fidelidad occidental", estableciendo, por ejemplo, sus primeros nexos comerciales con la Cuba castrista, una nación que, por decisión de la Organización de Estados Americanos (OEA), permanecía aislada en el concierto regional (solo México mantuvo su Embajada en La Habana, aunque muchos atribuyen esta decisión al deseo de Washington de conservar un "ojo" en su "patrio trasero").

Sobre la base de estos antecedentes, y en la línea de la tendencia historiográfica evocada en el primer párrafo, este artículo busca ofrecer una reflexión sobre las singularidades de la política internacional chilena durante los seis años de gobierno democratacristiano. La nueva proximidad establecida con la URSS y con los países del Este, así como el controvertido acercamiento con La Habana y la China maoísta, constituyen pruebas elocuentes de la voluntad de autonomía y de internacionalismo pragmático que caracterizó el proyecto reformista de Frei.

\section{Perforando la "cortina de hierro": la "Revolución en Libertad" ante el mundo del Este}

La reformulación de la relación de Chile con el mundo no es solo el producto de una adaptación a las circunstancias locales y mundiales. El establecimiento de "nuevas líneas de acción en las Relaciones Internacionales" (PARTIDO DEMÓCRATA CRISTIANO, 1967 , p. 53) fue una prioridad que figuraba desde el comienzo en el programa de la "Revolución en Libertad". En este sentido, las transformaciones sociales y económicas prometidas por las autoridades democratacristianas (reforma agraria, "promoción popular", nacionalización parcial de los recursos nacionales, sindicalización campesina, planes de vivienda, etc.) iban de la mano con el incremento de las conexiones exteriores, fenómeno que satisfacía una doble ambición: demostrar la existencia de una voluntad de independencia y multiplicar las fuentes potenciales de financiamiento para llevar a cabo los ambiciosos ejes programáticos definidos durante la campaña electoral. Esta convergencia entre formulación ideológica y satisfacción de objetivos pragmáticos fue enunciada por el diplomático falangista Enrique Bernstein, quien, reivindicando una "política exterior digna e independiente", consideraba que "ya no es posible que los países se constituyan en rivales peligrosos porque sustentan sistemas políticos [...] o concepciones filosóficas, religiosas o éticas que no son coincidentes". Comentando estas palabras, el órgano del PDC, Política y Espíritu, señalaba que, si bien "esta actitud se ve abonada por fuertes razones de tipo económico, sobre todo si consideramos que 
Chile es un país que depende en gran medida de las exportaciones", la "razón profunda de la decisión del Gobierno" nacía "de uno de los postulados más importantes de la doctrina demócrata cristiana" .En virtud de esta última, prosigue el texto, la postura del oficialismo

tiende a ampliar el radio de acción en lo interno y en lo externo, derrumbando murallas que parecían infranqueables y que separaban - y aún separan - a los seres humanos entre sí (Política Internacional De Chile, 1965, p. 6-7).

Más tarde, una publicación editada por el PDC en 1967 destacaba que "ninguno de los graves problemas que enfrenta Chile [...] puede ser resuelto cabalmente por el país concebido en forma aislada". De ahí que, "sin tapujos políticos", era indispensable que La Moneda buscara la ampliación de sus relaciones internacionales, "sin más limitaciones que las que impone el propio interés del país" (PARTIDO DEMÓCRATA CRISTIANO, 1967, p. 130) ${ }^{1}$.

Por otro lado, la decisión de abrir sedes diplomáticas en los países del Este representaba una auténtica ruptura para una nación que - salvo por un breve lapso en un contexto de prestigio creciente de la URSS debido a la acción del Ejército Rojo durante la Segunda Guerra Mundial (1944-1947) - no había mantenido relaciones oficiales con Moscú (a diferencia, por ejemplo, de Argentina y Uruguay). La renovada voluntad hacia la esfera socialista se reflejó tempranamente en la misión secreta de Enrique Bernstein, quien, por encargo directo de Eduardo Frei, debió dirigirse a Buenos Aires para reunirse con el Embajador soviético en el vecino país. A mediados de octubre de 1964, antes del inicio del nuevo mandato, el chileno le comunicó a su interlocutor, Alexander Alexeiev, los deseos del próximo Presidente de Chile. Sin poder esconder su satisfacción, el representante del Kremlin guardó sin embargo cierta mesura,recordando que debía consultarlo primero con sus superiores. Menos de

\footnotetext{
1 El esfuerzo transformador de la Democracia Cristiana fue incluso saludado por miembros de partidos de izquierda. En una carta dirigida a Orlando Letelier, Salvador Allende confesó que, en muchos aspectos, el PDC "habla un lenguaje próximo al nuestro", aunque, a juicio del futuro Presidente socialista, hacía falta una mayor "definición frente al imperialismo" (AMOROS, 2013 , p. 184). No obstante, en 1965, a raíz de la intervención estadounidense en República Dominicana, Allende no dudó en elogiar la postura de Eduardo Frei, quien, como veremos más adelante, condenó sin ambigüedades la injerencia de Washington (AMOROS, 2013, p. 213).
}

20 horas después del encuentro, Bernstein recibió en su hotel la precipitada visita de su colega soviético, siendo informado, como era previsible, del visto bueno de Moscú (BERNSTEIN, 1986, p. 173-175). Como esta positiva reacción nos lo deja entender, no cabe duda que para las autoridades de la URSS el gesto de La Moneda representaba una muy buena noticia.

En efecto, desde la segunda mitad de los años 1950 , la potencia socialista se había abocado con determinación a ampliar sus conexiones con la esfera occidental y las naciones del llamado "Tercer Mundo". Este renovado "internacionalismo", que contrastaba violetamente con los años de aislamiento estaliniano (RUPPRECHT, 2015, p.1-9), se expresó por medio de un visible acercamiento con naciones tales como la India de Jawaharlal Nehrú y el gobierno egipcio de Gamal Abdel Nasser, y tuvo una espectacular secuela en América Latina cuando en 1960NikitaJruschov resolvió socorrer a Cuba e iniciar una estrecha alianza con Fidel Castro que se prolongaría por tres décadas.

Sin embargo, al sur del mar Caribe las pretensiones soviéticas se estancaban. Si bien Uruguay y Argentina mantenían sus lazos oficiales con la URSS, esto se explicaba más bien por una inalterada tradición diplomática cuyos origines remontaban a los años 1930. Brasil, por su parte, también poseía una Embajada soviética en su territorio, pero con el derrocamiento de Joao Goulart a comienzos de 1964, quien no escondía su buena disposición hacia Moscú, las perspectivas de las relaciones bilaterales parecían menos prometedoras ${ }^{2}$. De ahí que la decisión de Frei constituyese una auspiciosa oportunidad, que anunciaba una integración regional y facilitaba la presencia creciente de las influencia soviéticas en el continente. Así lo constató el primer Embajador chileno en Moscú, Máximo Pacheco, quien, desde su llegada a la capital soviética en junio de 1965, recibió innumerables muestras de aprecio y un trato particularmente privilegiado. En sus profusos informes enviados durante los primeros años de misión diplomática, Pacheco subrayaba la cordialidad de sus interlocutores (se reunió con las autoridades más influyentes de la jerarquía soviética: Alekséi Kosygin, Leonid Brezhnev, Anastás Mikoyán, Andréi Gromyko y Nikolái Podgorni), así como el amplio

\footnotetext{
2 Pero, contrariamente a lo que podría pensarse, el establecimiento en Brasil de dictaduras militares anti-comunistas no detuvo el ritmo de los intercambios comerciales y culturales entre ambos países. Por el contrario, las convergencias económicas entre Brasil y la URSS incentivaron una amplia cooperación basada en consideraciones pragmáticas (TURRENT, 1986; RUPPRECHT, 2011).
} 
espacio ocupado por Chile en la prensa local, el realce de las celebraciones ligadas a su país (Fiestas Patrias, primer aniversario de la firma del restablecimiento de relaciones bilaterales, etc.), las reiteradas invitaciones para que el Presidente Frei visite Moscú o la entusiasta recepción de autoridades gubernamentales como el Ministro del Trabajo William Thayer ${ }^{3}$. Según James Holger, Primer Secretario de la Embajada en Moscú,

a juzgar por los comentarios de [...] corresponsales soviéticos y extranjeros, nuestro país era, en esos años, el que contaba con la mayor acogida política y económica, después de Cuba, por parte del Gobierno de la URSS [en América Latina] (PACHECO y HOLGER, 2009, p. 146).

En un informe elocuente fechado en enero de 1966, Pacheco se dirigía personalmente a Gabriel Valdés (y no al Ministerio de RR.EE. como el protocolo exige) para insistir en las ventajas ofrecidas por la cooperación con Moscú, cuyos dirigentes le habían expresado "en forma reiterada el deseo de la URSS de estrechar relaciones culturales y económicas". No dudaba en calificar el "ambiente político" percibido como "espléndido", pero advertía que para obtener beneficios de la asistencia soviética había llegado "la hora de la decisión, la cual no puede retardarse ni evitarse" (AHMAEC, Máximo Pacheco a Gabriel Valdés, 14 ene. 1966). No debe extrañarnos que para azuzar la consolidación de los lazos con la URSS el Embajador chileno haya optado esta vez por enviar su misiva directamente al Ministro Valdés, quien era, además, su amigo cercano. Si bien la aceleración de los lazos con el Este era un objetivo explícito del programa de las autoridades, el positivo transcurso de las relaciones debe mucho a la voluntad del Canciller. Fue este último quien efectuó el discurso en el acto de formalización de la apertura de relaciones, revelando en su alocución el carácter tanto ideológico

\footnotetext{
3 Todos estos antecedentes evocados en los informes del Embajador Máximo Pacheco pueden ser consultados en el Archivo Histórico del Ministerio de Asuntos Exteriores de Chile (Fondo "Países - Rusia"), donde los responsables, a quienes agradezco profundamente, suelen brindar una excepcional recepción a los investigadores. Véase (AHMAEC, Máximo Pacheco al Ministro de Asuntos Exteriores de Chile, 8 jun. 1965; AHMAEC, Máximo Pacheco al Ministro de Asuntos Exteriores de Chile, 6 de jul. 1965; AHMAEC, Máximo Pacheco al Ministro de Asuntos Exteriores de Chile, 12 jun. 1965; AHMAEC, Máximo Pacheco al Ministro de Asuntos Exteriores de Chile, 21 sept. 1965; AHMAEC, Máximo Pacheco al Ministro de Asuntos Exteriores de Chile, 27 jun. 1966; AHMAEC, Máximo Pacheco al Ministro de Asuntos Exteriores de Chile, 28 sept. 1966; AHMAEC, Máximo Pacheco al Ministro de Asuntos Exteriores de Chile, 21 sept. 1967).
}

como pragmático de la decisión. En presencia del Embajador de la URSS en Buenos Aires, Valdés se preocupó por dejar de manifiesto sus inclinaciones favorables hacia el nuevo socio:

El Gobierno de Chile considera que sus relaciones normales con la Unión de Repúblicas Socialistas Soviéticas contribuirán a afianzar la paz y la cooperación pacífica de todos los pueblos; estima esenciales estas relaciones para dar mayor amplitud a nuestro comercio exterior [...] Estamos seguros que ellas serán fructíferas y, más allá de la forma diferente de los regímenes políticos, habrá una cooperación entre nuestro pueblo, generoso y abierto, amante de sus tradiciones y de sus libertades, y el gran pueblo ruso, cuyos sufrimientos en la guerra, cuya bondad y cuyo amor por la paz son una parte del gran patrimonio de la humanidad entera (Memoria del Ministerio de Relaciones Exteriores correspondiente al año 1964, p. 44).

El uso del adjetivo "ruso", en vez de "soviético", constituye un signo a tener cuenta. Al emplear dicha expresión, el Ministro busca reiterar su disposición hacia la sociedad de la URSS y su admiración por sus raíces históricas, pero distanciándose sutilmente de su identidad ideológica, forjadora de la creación del Estado "soviético" en 1922. Es una hábil estrategia retórica destinada a evitar eventuales reticencias internas, en un país donde los prejuicios hacia la "órbita comunista" eran intensos. El titular de RR.EE., convencido de la necesidad de ampliar los nexos con el Este, revelaba así su apertura, sin que fuese posible reprocharle un distanciamiento respecto al campo occidental, al que Chile pertenecía y seguiría perteneciendo, aunque desde ahora con un mayor margen de independencia.

Como lo recuerda hoy en día su viuda Sylvia Soublette, ella y su marido mantenían una estrecha relación de amistad con el primer Embajador soviético en Santiago, Alexander Anikin, lo cual incluso -siguiendo las confesiones de Soublette- generó un cierto malestar de parte de un representante diplomático norteamericano (Entrevista con Sylvia Soublette, Santiago, 25 oct. 2016). Otro indicio de la favorable posición de Gabriel Valdés respecto a la URSS quedó en evidencia cuando el 1으 de agosto de 1966, y luego de un violento ataque verbal dirigido por Fidel Castro a Eduardo Frei en su tradicional mensaje del 26 de julio, el Ministro se precipitó para comunicarle a Máximo Pacheco las instrucciones a seguir. A pesar de la virulencia del aliado caribeño del Kremlin, 
quien "injurió burdamente a nuestro Presidente", y del beneplácito otorgado a Fidel Castro por los dirigentes chilenos del Partido Socialista y Comunista, era preciso "manifestar al Gobierno soviético que esta acción no modificará nuestro criterio de continuar ampliando y profundizando las excelentes relaciones que tenemos" (AHMAEC, Gabriel Valdés a Máximo Pacheco, 1 ago. 1966).

La voluntad de Valdés hacia Moscú se acompañó también de una actitud crítica y, por momentos, desafiante hacia la política exterior de la Casa Blanca, lo que suscitó la recriminación de su superior, Eduardo Frei, y el disgusto de los representantes diplomáticos norteamericanos en Santiago, quienes criticaban la "lógica gaullista" adoptada por el Canciller (HENRÍQUEZ y VAN KLAVEREN, 2016, p. 360). Luego de convocar a una conferencia de Ministros de Relaciones Exteriores de todo el continente, reunidos en Viña del Mar en mayo de 1969 bajo la autoridad de la CECLA (Consejo Especial de Coordinación Latinoamericana), los delegados redactaron una resolución que el propio Valdés se encargó de presentar más adelante al Presidente Richard Nixon en la Casa Blanca. En presencia de Henry Kissinger, Consejero de Seguridad Nacional, el chileno subrayó los beneficios excesivos que los Estados Unidos obtenían de sus inversiones en América Latina, una observación que generó la incomodad del Jefe de Estado y la visible molestia de Kissinger. En respuesta, este último citó al Ministro a almorzar, dando lugar a un encuentro particularmente tenso durante el cual, según cuenta Valdés en sus memorias, llegó incluso a catalogar de "imperialistas" las reflexiones de su interlocutor (VALDÉS, 2009, p. 196).

Más allá de este incidente, en términos generales, la administración democratacristiana supo desde un comienzo hacer oír su postura en torno a las relaciones interamericanas $\mathrm{y}$, en ocasiones, distanciarse de las medidas adoptadas por Washington. Un buen ejemplo de ello quedó de manifiesto durante la ardua controversia avivada a raíz de la intervención del Cuerpo de Marines de los Estados Unidos en República Dominicana, un país donde el Presidente reformista Juan Bosch parecía estar amenazando los intereses del gobierno liderado por Lyndon Johnson. La reacción de la administración Frei fue inmediata y decidida: el Ministro Valdés denunció la intervención, calificándola como una violación de los principios de la OEA, e hizo un llamado a la organización continental a condenar las acciones de la "potencia del Norte" (HURTADO, 2016, p. 100).
Mientras que las diferencias entre la postura de $\mathrm{La}$ Moneda y la doctrina intervencionista de su tradicional aliado, los EE.UU., se hacían visibles, el gobierno del PDC seguía fortaleciendo sus vinculaciones con el "mundo socialista". Esta tendencia fue confirmada en el transcurso del año1965, cuando el Ministerio de Relaciones Exteriores anunció el establecimiento de lazos diplomáticos con cinco "Democracias Populares": Hungría, Checoslovaquia, Bulgaria, Rumania y Polonia (Memoria del Ministerio de Relaciones Exteriores correspondiente al año 1965, p. 163). Dos años más tarde, el acercamiento con la URSS comenzó a producir resultados concretos, como los tres acuerdos de cooperación técnica-comercial firmados en enero de 1967por el Embajador Pacheco, con lo cual, después de Cuba y de Brasil, Chile se transformaba en el tercer país latinoamericano en suscribir este tipo de protocolos con Moscú (PACHECO y HOLGER, 2009, p. 55).De forma simultánea, los representantes diplomáticos de ambas naciones se esmeraban en negociar un tratado en el rubro de la pesca, así como un plan de colaboración para la instalación en Chile de una fábrica de casas prefabricadas (CLISSOLD, 1970, p. 206) ${ }^{4}$.

Algo similar sucedía con los demás países del Este con los cuales Chile había intercambiado misiones diplomáticas, destacándose, por ejemplo, la intensa actividad bilateral con las autoridades rumanas. No solo ambos Estados suscribieron tres convenios en 1968, incluido un tratado de cooperación cultural, sino que ese mismo año dos importantes representantes del gobierno de Nicolae Ceausescu aterrizaron en Santiago para cumplir una misión oficial: el titular de Relaciones Exteriores, Cornelio Manescu, y su colega de la cartera de Educación (Memoria del Ministerio de Relaciones Exteriores correspondiente al año 1968, p. 7-8, 28-29, 205-213) $)^{5}$. A finales de febrero 1970, el Ministro de Minería del PDC, Alejandro Hales, anunció a la prensa la constitución de una sociedad mixta chilenorumana inaugurada con el fin de explorar y explotar los minerales polimetálicos de la provincia de

\footnotetext{
4 Estos dos acuerdos, llevados finalmente a cabo en tiempos de la Unidad Popular (1970-1973), han sido generalmente presentados como manifestaciones de "solidaridad ideológica" hacia el gobierno de Salvador Allende. Vemos, sin embargo, que la voluntad de la URSS de ofrecer asistencia a Chile era ya una situación patente en los años anteriores, lo que debiera invitarnos a analizar la evolución de los lazos Chile-URSS bajo una óptica de continuidad y no como un proceso que experimentara una ruptura radical en 1970, año del triunfo electoral de Allende.

5 Podríamos haber evocado otros ejemplos de países del Este que estrecharon sus vínculos con el gobierno del PDC. El caso de Checoslovaquia ha sido ampliamente estudiado por Michal Zourek (ZOUREK, 2014).
} 
Atacama, en el norte del país. Los delegados rumanos, particularmente agradecidos hacia la disposición de La Moneda, auguraron que este proyecto conjunto "será un buen punto de partida para otras iniciativas y empresas de gran futuro"(Clarin, Santiago, 25 feb. 1970, p. 4).

En un ámbito más simbólico, la mayor cercanía que rápidamente se estaba produciendo entre Chile y el mundo del Este tuvo un visible impacto en la esfera cultural e intelectual. Si bien antes de 1964, los artistas que solían atravesar la "cortina de hierro" eran mayoritariamente militantes activos de la izquierda chilena -como Pablo Neruda y Francisco Coloane- o figuras cercanas al Partido Comunista - como Margot Loyola y Violeta Parra-, a partir de mediados de la década de 1960 los perfiles tendieron a diversificarse. En un contexto de apertura política, múltiples e inesperadas personalidades aterrizaron en Moscú para presentarse ante el público soviético, a pesar de sus evidentes aprehensiones ideológicas. Es el caso del conjunto folklórico, conocido por su ulterior apoyo a la dictadura de Augusto Pinochet, "Los Huasos Quincheros", cuyos cuatro miembros tuvieron la posibilidad de conoceren 1966 más de 20 ciudades soviéticas, ofreciendo conciertos masivamente concurridos (AHMAEC, Máximo Pacheco al Ministro de Asuntos Exteriores de Chile, 8 feb. 1967, Entrevista con Benjamín Mackenna, Santiago, 11 ene. 2013).El Conjunto de Música Antigua de la Universidad Católica, dirigido por la compositora y esposa del Ministro Valdés, Sylvia Soublette, también recorrió las repúblicas socialistas durante una gira extenuante que incluyó las regiones del Báltico (Riga, Vilna, Tallin), la capital Moscú (donde fueron recibidos en el Kremlin por la Ministra de Cultura,EkaterinaFurtseva, quien les propuso grabar un disco al día siguiente) y Leningrado(actualmente San Petersburgo) para posteriormente desplazarse hacia Rumania y Yugoslavia(AHMAEC, Máximo Pacheco al Ministro de Asuntos Exteriores de Chile, 17 feb. 1967; Entrevista con Sylvia Soublette. Santiago, 25 oct. 2016$)^{6}$.

\footnotetext{
${ }^{6}$ De entre los múltiples ejemplos de artistas no comunistas que viajaron a la URSS en tiempos de Frei (Flora Guerra, Nicanor Parra, Malucha Solari, Roberto Bravo, Pedro d'Andurain, etc.),hagamos alusión a la aclamada tournée de Claudio Arrau, organizada a raíz de la explícita voluntad de los organizadores soviéticos. Máximo Pacheco inició las gestiones que desembocaron en 1968 en una gira por Kiev, Leningrado y Moscú. La prensa soviética no permaneció indiferente ante la presencia del maestro chileno, quien fue agasajado por el compositor Aram Jachaturián durante una cena en su honor (AHMAEC, Óscar Pinochet de la Barra al Ministro de Asuntos Exteriores, 10 jun. 1968).
}

Otra sorprendente delegación de chilenos aterrizó en Moscú en mayo de 1967 producto de una invitación cursada por el Ministerio soviético de Educación Superior: se trataba del conjunto de los integrantes del Consejo de Rectores (a excepción del titular de la Universidad de Chile, quien se excusó por razones de índole personal), incluida la autoridad máxima de la Universidad Católica, el Arzobispo, conocido por su conservadurismo, Alfredo Silva Santiago. Luego de evaluar las posibilidades de cooperación académica con instituciones de Moscú, Leningrado, Ereván, Kiev y la ciudad siberiana de Novosibirsk, los facultativos prosiguieron su larga misión de dos meses trasladándose a Polonia, Checoslovaquia y Hungría (AHMAEC, Máximo Pacheco al Ministro de Asuntos Exteriores de Chile, 31 mayo 1967; El Siglo, 27 abr. 1967, p. 4).

Estos son solo algunos ejemplos de los muchos que pudimos haber mencionado y que demuestran convincentemente que después de 1964 el mundo del Este se hacía cada vez menos inaccesible para sectores tradicionalmente desligados de las organizaciones prosoviéticas. La pretensión de multiplicar los vínculos a escala global, no solo contribuyó a acrecentar las conexiones políticas con la "esfera socialista", sino que permitió igualmente una mayor presencia de la cultura soviética y, en consecuencia, una paulatina familiarización con un mundo que permanecía, hasta ese momento, envuelto en un velo de misterio. Así, al emprender un programa más autónomo, basado en consideraciones prácticas y en la reivindicación de un nuevo proyecto ideológico, la "Revolución en Libertad" promovió una nueva etapa de transformaciones internas y globales, cuyas secuelas se hicieron visibles tanto en la esfera geopolítica como en el plano social.

\section{De la "Política de sangre sin Revolución" a la "la liberación del yugo imperialista": el proyecto de Eduardo Frei a los ojos del gobierno castrista}

En un primer momento, el evidente estrechamiento de los vínculos entre el gobierno de Frei y la URSS no fue bien percibido por el principal aliado latinoamericano de Moscú: la Revolución Cubana. En un contexto de tensión creciente entre los "barbudos" y los dirigentes del Kremlin, marcado por la profunda desilusión de Fidel Castro por su marginalización en las negociaciones que permitieron resolver la Crisis de los Misiles a fines de 1962, todo acercamiento 
entre la esfera socialista y los "regímenes burgueses" del continente era visto como un signo de debilidad ideológica. Si por un lado, Moscú, amparado bajo el principio de la "coexistencia pacífica", planteaba la necesidad de profundizar los lazos con el mundo occidental para evitar una catástrofe planetaria, Cuba, por su parte, pregonaba una estrategia revolucionaria basada en la lucha armada clandestina ${ }^{7} y$, por consiguiente, en la ruptura total e irrestricta con las estructuras vigentes. En este sentido, y a pesar de su carácter reformista, la "Revolución en Libertad" de Eduardo Frei no constituía, a los ojos del castrismo, una experiencia liberadora.

Por el contrario, como lo hemos evocado, el esfuerzo por presentar el "camino chileno" por parte de los estrategas de la OTAN y de la Casa Blanca como una alternativa democrática a las transformaciones del castrismo, irritó profundamente a los líderes de la Isla. El propio Fidel Castro lo reconocería al subrayar con visible ironía que "Los imperialistas han querido convertir la llamada 'experiencia chilena' en una experiencia para rivalizar con Cuba" (CASTRO, 1966, p. 16). Resultaba, por ende, indispensable atacar duramente el proyecto democratacristiano para despojarlo de toda "legitimidad revolucionaria" y así evitar que atraiga las simpatías de la izquierda latinoamericana. Cuando a comienzos de 1966 se produjo la mortal represión de los mineros cupríferos de El Salvador a manos de tropas del Ejército movilizadas por el gobierno, Castro no dudó en lanzar sus dardos envenenados contra Frei. Ante una delegación de parlamentarios chilenos, el Primer Ministro se preguntó primero: “¿Es que acaso está teniendo lugar una revolución?”, para luego profundizar en su predecible respuesta: "No. La primera gran falsedad en la que incurren estos intentos es en la creencia de la posibilidad de conciliar los intereses de clase, creer que se puede hacer una Revolución [...] con un espíritu de conciliación de clases". Y finalmente reservó una serie de punzantes epítetos para denostar al Presidente de Chile:

Frei es un reaccionario; Frei ha demostrado con este hecho ser no un individuo de mano firme, sino un

\footnotetext{
7 Más allá de los múltiples discursos de Fidel Castro (que ofrecen diversos, aunque por momentos contradictorios, elementos para entender las orientaciones de los cubanos), el mejor ejemplo de esfuerzo por teorizar la doctrina revolucionaria de los "barbudos" es la obra de Ernesto Guevara, La Guerra de Guerrillas. Más tarde, la tesis guevarista del "foquismo" sería retomada, profundizada y radicalizada por el filósofo francés y colaborador cercano de Castro, Régis Debray (DEBRAY, 1967).
}

individuo cobarde que abusa del poder, que lanza las tropas contra los obreros; Frei ha demostrado ser un mentiroso; Frei ha demostrado ser un político vulgar [...]; Frei demuestra al pueblo de Chile y al mundo qué tipo de revolución es la que va a hacer, $[\ldots]$ no una revolución sin sangre, sino una política de sangre sin revolución. ¡Sangre sin revolución! Esa es la política de Frei (CASTRO, 1966, p. 16).

Simultáneamente, mientras que el apoyo cubano a las guerrillas de América Latina comenzaba a transformarse en una fuente importante de fricción entre La Habana y Moscú -y, por extensión, los Partidos Comunistas fieles a las orientaciones emanadas de la URSS-atacar al gobierno democratacristiano constituyó un medio sutil de recriminar ala administración de Leonid Brézhnev. Así quedó confirmado en 1966 durante la tradicional alocución del 26 de julio, cuando el "Comandante" recibió una ovación por dejar al descubierto sus reparos respecto a la política soviética hacia Chile: "nosotros los cubanos nos consideramos con todo el derecho a sentirnos agraviados, [...] heridos con cualquier país que le brinde al régimen de Frei cualquier asistencia técnica y económica" (Granma: résuméhebdomadaire, La Habana, 31 jul. 1966, p. 11). El discurso castrista, como bien lo percibieron los expertos de la OTAN, incomodaban fuertemente al Kremlin, que intentaba apaciguar los ánimos insistiendo en la doctrina de no-intervención (Archivo OTAN, C-M(66)39, 11 mayo 1966, p. 8). Un paso adicional fue franqueado en marzo de 1967 cuando nuevamente Fidel Castro evidenció sus diferencias con la política continental de Moscú. Aludiendo a Frei y al Presidente de Colombia, Carlos Lleras Restrepo, quien también había iniciado un acercamiento con la URSS, el "Líder Máximo" recordó que Cuba no establecería "relaciones diplomáticas hasta tanto no sean gobiernos revolucionarios los que dirijan a esos países". Se trataba de un indiscutible reproche dirigido a Moscú. Así fue interpretado por el Embajador belga George Elliott, quien además evocó la existencia de una serie de rumores en La Habana según los cuales Fidel Castro habría dicho que sería "un error creer que Cuba no puede vivir sin la URSS" y que, "sobre una base exclusivamente comercial", "preferiría infinitamente negociar con Europa occidental"(Archivos del Reino de Bélgica, George Elliott a Pierre Harmel, 16 marzo 1967).

Estas tensiones se agudizaron hasta alcanzar su paroxismo en agosto de 1967 durante la famosa Conferencia de la Organización Latinoamericana de Solidaridad (OLAS). En esta ocasión, no solo la 
gran mayoría de los delegados avalaron el llamado decidido de los cubanos a emprender la lucha armada en el continente, sino que también firmaron una resolución condenando "ciertos países socialistas" por colaborar financieramente con "regímenes contrarevolucionarios" (LÉVESQUE, 1976, p. 153-154). Claro está que dentro de esos aludidos "países socialistas" se apuntada a la URSS y que por "regímenes contra-revolucionarios" debía entenderse, entre otros, el gobierno de Eduardo Frei. Observamos, por ende, que las vicisitudes de las relaciones entre la Revolución Cubana y la potencia del Este permanecían íntimamente ligadas a la postura hacia La Moneda, articulando así una lógica transnacional que ameritaría ser profundizada. En la medida en que los lazos con Moscú se deterioraban, las críticas hacia la administración del PDC, dispuesta a ampliar sus vínculos con el Este, se exacerbaron.

Siguiendo esta lógica conectada, no debiera sorprendernos que el paulatino proceso de "normalización" de las relaciones Cuba-URSS, visible a partir de finales de 1968, haya estado acompañado de una notoria atenuación del tono agresivo empleado por La Habana. Pero la nueva disposición del castrismo hacia Chile está igualmente asociada a un conjunto de gestos efectuados por el gobierno de Frei. ¿Cómo es posible que un gobierno calificado en un primer momento con los peores epítetos termine mereciendo la venia de los cubanos? ¿O que incluso Fidel Castro le haya recomendado a Salvador Allende mantener en sus funciones a Gabriel Valdés ${ }^{8}$, Ministro de un Presidente anteriormente considerado como un "reaccionario" y un "mentiroso"?

Como lo recuerda el periodista y ex militante comunista Eduardo Labarca, el episodio que inició el aplacamiento de la crítica castrista hacia la administración democratacristiana fue la posición de Frei ante el ruidoso "caso de los guerrilleros del Che" (LABARCA, 2014, p. 235). En efecto, después de la represión que acabó con la vida de Ernesto Guevara en Bolivia, tres sobrevivientes cubanos lograron escapar, siendo sin embargo detenidos en territorio chileno.

\footnotetext{
8 Esto es al menos lo que sugiere un documento soviético (ALEKSEEV, 1998, p. 413).En una entrevista que nos fue concedida, el ex diputado del Partido Radical, Camilo Salvo, quien acudió a los festejos del 26 de julio de 1970 en La Habana en medio de la campaña presidencial de Allende, corroboró esta información. Según Salvo, sus interlocutores cubanos "creían primero que perdíamos la elección, estaban convencidos que perdíamos", pero estimaban que mediante "un entendimiento con la Democracia Cristiana" las posibilidades de un triunfo electoral serían mayores (Entrevista con Camilo Salvo, Santiago, 5 sept. 2016).
}

Luego de una serie de tratativas diplomáticas con los países vecinos, el Canciller Valdés constató con estupefacción que ni Perú, ni Argentina facilitarían el paso de los combatientes para ser devueltos a Cuba. La solución adoptada pasó finalmente por un acuerdo con Francia, país que aceptó recibir a los tres guerrilleros para luego -vía Isla de Pascua, Tahití y París- ser enviados de regreso a La Habana.

Pero más allá de este hecho preciso, cuyas consecuencias positivas podrían haberse difuminado con el tiempo -como ha sucedido tantas veces en las inestables relaciones mantenidas por Fidel Castro con distintos gobiernos del continente-, el argumento esencial que favoreció la estabilización de los lazos entre Santiago y La Habana fue, sin lugar a dudas, la decisión de Chile de restablecer los intercambios comerciales con la Isla a pesar de la política de aislamiento decretada en la Conferencia de Cancilleres de Washington en julio de $1964^{9}$. Ofreciendo una primera esperanza para la reintegración regional del gobierno castrista, la administración democratacristiana autorizó en febrero de 1970 la firma de un ambicioso y polémico convenio comercial con Cuba. Este protocolo, vilipendiado por la derecha más intransigente y ensalzado por los partidos de izquierda, fue la culminación de un largo proceso iniciado un año y medio atrás y que explicaba en gran parte la inesperada armonía que caracterizó las relaciones chileno-cubanas a lo largo de los dos últimos años de Eduardo Frei.

Como los antecedentes expuestos en este apartado nos lo demuestran, el Chile de Frei constituía un buen barómetro para evaluar el nivel de las disensiones Cuba-URSS. Hemos observado que la aplicación de una perspectiva global y conectada, orientada al examen de los fenómenos internacionales en clave multilateral, nos permite entender de mejor manera la hostilidad, así como la posterior estabilización, de las relaciones entre La Habana y Santiago, ligadas, en gran medida, a las vicisitudes de la alianza cubano-soviética.

\section{Las relaciones comerciales: un primer paso hacia la integración regional y mundial (China y Cuba)}

Fue el joven militante del PDC, Belisario Velasco, gerente comercial de la Empresa de Comercio

\footnotetext{
Sobre la difícil decisión que tuvo que asumir el gobierno de Jorge Alessandri (1958-1964) de suspender las relaciones diplomáticas y comerciales con Cuba en 1964, véase el completísimo artículo de Joaquín Fermandois (FERMANDOIS, 1982).
} 
Agrícola (ECA), quien inició las negociaciones con Cuba. Siguiendo "una resolución del Presidente de la República y de Gabriel Valdés", según la cual "Chile debía tener relaciones con todos los países del mundo si le conviniera" (Entrevista con Belisario Velasco, Santiago, 20 sept. 2016), Velasco se dirigió en un primer momento a China en marzo-abril de 1968. Destacamos esta primera misión, ya que la voluntad de ampliar los lazos económicos con la potencia asiática, con la cual no se poseían relaciones diplomáticas, revela nuevamente la apertura del gobierno chileno, así como su pragmatismo a la hora de evaluar los intereses nacionales. En efecto, el viaje a China respondía a un impase con Buenos Aires, que no estaba cumpliendo con un contrato de celulosa establecido con Chile. La solución hallada por el Ministro Valdés para "estudiar una contramedida que hiciera comprender [...] que los contratos no podían violarse sin costos", fue suspender las importaciones de té al gobierno argentino y compensar esa carencia mediante una compra masiva en China. Así fue como Belisario Velasco pudo recorrer Pekín y sus alrededores, entrevistarse con Mao Tse Tung, efectuar un discurso ante cientos de miles de chinos reunidos en homenaje a Martin Luther King, recientemente asesinado, y, por último, firmar un contrato por mil toneladas de té (VALDÉS, 2009, p. 181; Entrevista con Belisario Velasco, Santiago, 20 sept. 2016).

El éxito que coronó la estadía de Velasco en tierra asiática es una prueba de que La Moneda en tiempos de Frei, sin negar su pertenencia a la esfera occidental, estaba dispuesta a mirar hacia los países socialistas con el objeto de encontrar soluciones a sus necesidades prácticas. Algo similar se produjo pocas semanas más tarde, cuando el mismo Belisario Velasco aceptó representar a su país en La Habana para negociar un inédito intercambio comercial. Esta decisión, como lo hemos evocado, no estaba exenta de dificultades. Con ella la Democracia Cristina optaba por romper el tradicional bloqueo votado por las naciones del continente en 1964. Enviar productos a Cuba representaba, en efecto, un desafío al orden interamericano y a la casi inalterable fidelidad de esos países hacia la política hemisférica de los Estados Unidos. Por otra parte, esta decisión reflejaba el correcto equilibrio entre reformismo y pragmatismo que el programa gubernamental intentaba poner en práctica. Como lo reconoce Rafael Moreno, Vice-Presidente de la Cooperativa de Reforma Agraria (CORA), uno de los objetivos de la operación consistía en exportar ciertos productos (cebollas, ajos, porotos) que "habían resultado difíciles de colocar en los mercados internos", una táctica respaldada explícitamente por el Presidente Frei (MORENO, 2014, p. 541). De esta manera, el gobierno "mataba dos pájaros de un tiro", al reafirmar los postulados de su postura diplomática ofreciendo, simultáneamente, una prueba tangible de los beneficios generados por la controvertida Reforma Agraria. Como lo recuerda el principal artífice de este proceso, la estrategia de "abrirse a las exportaciones" permitía "enfrentar la crítica de que la Reforma Agraria producía una caída de la producción" (Entrevista con Rafael Moreno, Santiago, 6 mar. 2017). La reanudación de los intercambios agrarios con Cuba se inscribía, por ende, en una lógica doble: una estrategia internacional - marcada por la apertura hacia territorios antes desestimados- y otra interna, destinada a legitimar los objetivos programáticos de la administración Frei.

Antes del anuncio oficial de la reanudación de las exportaciones a Cuba, en febrero de 1970, el intercambio comercial se reducía a una modesta cantidad de vino que, gracias a sus contactos personales con las autoridades de la Isla, el senador de izquierda, Baltazar Castro, hacía llegar a La Habana (DRAGO, 1971, p. 13). A pesar de la mala reputación de "Don Balta", el brebaje producido en la viña El Rosario (Rancagua), el político chileno sin embargo se esmeraba en publicitar sus gestiones, a través, por ejemplo, de un curioso concurso literario llamado a exaltar la "amistad chileno-cubana" (Vino chileno para Cuba: Antología poética, 1972). Más allá de esta efímera exportación, reemplazada en tiempos de Salvador Allende por botellas de mejor calidad (Entrevista con Francisco Fernández, Santiago, 16 nov. 2016), ningún otro producto nacional acostaba los puertos del país caribeño.

En este contexto, Belisario Velasco, luego de su largo periplo en Cuba donde gozó de la compañía constante de Fidel Castro ${ }^{10}$, logró establecer precios de venta bastante favorables para Chile y que desembocarían en el envío de porotos, cebollas y ajos chilenos durante el año 1970. Según lo que nos señalara el propio Belisario Velasco, quien estaba consciente de la inevitable controversia que sus gestiones azuzarían en su país, en su primera estancia

\footnotetext{
${ }^{10}$ Poco antes de la misión de Belisario Velasco, Baltazar Castro, durante uno de sus viajes a La Habana y con la venia de las autoridades democratacristianas, ya había tenido la posibilidad de comunicarle a Fidel Castro que el gobierno de Chile estaba "de acuerdo en que había que realizar algo positivo para normalizar las relaciones de los países americanos respecto a Cuba". La idea era evaluar la disposición del "Líder Máximo" antes de emprender cualquier acción en este sentido (CASTRO, 1969, p. 121).
} 
habanera le sugirió al "Líder Máximo" que la Iglesia de la Isla se manifestara públicamente a favor del acercamiento comercial interamericano, lo que sin duda minimizaría la hostilidad de la derecha y, en el caso de Chile, ofrecería a Frei una mayor legitimidad a la hora de anunciar la polémica decisión. Así fue cómo en abril de 1969 la Conferencia Episcopal de Cuba, que en la práctica poseía un poder apenas simbólico, publicó un folleto de cuatro páginas mediante el cual los signatarios denunciaban "el aislamiento en que venimos viviendo", "las dificultades externas", así como "las estructuras contemporáneas de las relaciones entre los pueblos injustamente desventajosas para los países débiles". Luego de aclarar estos principios, los prelados encabezados por el Arzobispo de La Habana, apuntaban directamente a los perjuicios del bloqueo económico y efectuaban un elocuente llamadoa poner fin a esta práctica, "cuya prolongación automática acumula graves inconvenientes a nuestra Patria":

Buscando el bien de nuestro pueblo y fieles al servicio de los más pobres conforme al mandato de Jesucristo y al compromiso proclamado nuevamente en Medellín denunciamos esta injusta situación de bloqueo que contribuye a sumar sufrimientos innecesarios y a hacer más difícil la búsqueda del desarrollo. Apelamos, por tanto, a la conciencia de cuánto están en condiciones de resolverla para que emprendan acciones decididas y eficaces destinadas a conseguir el cese de esta medida (EVELIO et al., 1969).

No cabe duda que ante esta decidida declaración, indisociable de la voluntad anunciada por los dirigentes chilenos, el gobierno del PDC podría actuar con mayor libertad, amparado en los argumentos morales que la declaración de la Iglesia cubana había puesto sobre la mesa. Por lo demás, el comunicado de los obispos parece haber tenido una cierta difusión en los medios locales. El periódico de izquierda, Clarín, al anunciar que Chile "romperá el bloqueo", reconocía que las "quejas" eran inevitables, pero que ante ellas, se podrían evocar las "razones humanitarias [...] por cuanto una declaración de los obispos de la Isla señala que es necesario llevar a La Habana ese tipo de alimentos que precisan los niños en su crecimiento" (GAMBOA, 1970, p. 3). Estas mismas "razones humanitarias" serían recordadas por el Canciller Gabriel Valdés durante una conferencia de prensa, en la cual negaba estar violando los acuerdos de la OEA debido a que los productos exportados "formaban parte de la dieta normal y necesaria de la población" (VALDÉS, 2009, p. 184-185), un principio legalmente poco convincente, pero que podría generar una mayor receptividad de la opinión pública.

El comunicado episcopal constituye uno de los tantos factores destinados a acallar la oposición interna. Pero Belisario Velasco contaba también con otro astuto argumento. Era necesario, a su juicio, convencer a los agricultores del valle central de Chile de participar en las transacciones. Estos últimos, a pesar de su reconocido conservadurismo, privilegiaron en efecto el pragmatismo económico y aceptaron jugar un rol importante en el envío de alimentos a Cuba. El primero en aceptar fue Benjamín Matte, Presidente de la Sociedad Nacional de Agricultura (SNA), "la flor y nata de la derecha chilena" (Entrevista con Belisario Velasco, Santiago, 20 sept. 2016), quien disponía además de terrenos cultivados en la zona central del país. Entrevistado más tarde por el periódico Las Noticias de Última Hora, el empresario destacó las "ventajas evidentes" del acuerdo y recordó que Cuba "objetivamente es un mercado que presenta seguridad para la producción y la exportación”. Luego de indicar que la anterior ausencia de relaciones comerciales con la Isla obedecía a "políticas equivocadas", Matte acentuó los innegables beneficios que brindaban las exportaciones: "Considere que el monto de la operación es del orden de los 30 millones de dólares. Es decir, una cifra igual al total de las exportaciones agropecuarias chilenas durante 1969"(Las Noticias de Última Hora, Santiago, 9 agosto 1970, p. 6). Los lazos entre la SNA y ALIMPORT, la empresa estatal de Cuba que ejecutaba las transacciones agrícolas internacionales, se fortalecieron en el trascurso del año 1970. No solo los terratenientes chilenos recibieron a un grupo de delegados cubanos en las dependencias del aristocrático Hotel Crillón a mediados de año, sino que incluso enviaron una delegación encabezada por el propio Matte a La Habana (Las Noticias de Última Hora, Santiago, 15 de jul. 1970, p. 2).

Las negociaciones de Belisario Velasco durante los años 1968 y 1969, astutamente dirigidas a asegurar la presencia de empresarios de derecha, desembocaron en la oficialización de un protocolo oficial. Fue Rafael Moreno quien asumió la responsabilidad de anunciar, el 20 de febrero de 1970, la venta por tres millones de dólares a Cuba, un operativo que contaría tanto con la participación de los asentamientos campesinos formados tras la extensiva reforma agraria impulsada por el gobierno democratacristiano y gestionada por el propio Moreno desde la CORA, como con la de "agricultores particulares" (El Mercurio, Santiago, 
21 feb. 1970, p. 1). Las críticas hacia la iniciativa gubernamental, tanto en el escenario interno como en el sistema internacional, no se hicieron esperar, a pesar de que la injerencia de estos "agricultores particulares" disminuía el impacto de la reacción de los detractores. FIDUCIA (Sociedad Chilena de Defensa de la Tradición, Familia y Propiedad), una organización ultra-católica vinculada a las capas altas y medios universitarios, acusó ala SNA de traicionar los intereses de la nación (Las Noticias de Última Hora, Santiago, 9 ago. 1970, p. 6). El diario más representativo de la derecha, El Mercurio, se sumó a las acusaciones contra la Cancillería, condenando un "movimiento de reconciliación generado unilateralmente y en cuya virtud se perdonan los antiguos agravios, se niega que Castro represente un peligro para la vida institucional". A juicio de este órgano, el gesto oficial equivalía a "confesar [...] que no corresponde al régimen castrista dar un paso hacia América Latina sino que, por el contrario, serían los países de ésta los obligados a recuperar la amistad de La Habana". En el mismo artículo, el periodista reprodujo también la opinión hostil del Ministro de Relaciones Exteriores de Colombia, Alfonso López Michelsen, demostrando así que la polémica había trascendido largamente la esfera local (El Mercurio, Santiago, 21 feb. 1970, p. 3).Como era de esperarse, Washington tampoco se mantuvo al margen y el Embajador estadounidense llamó airado al Presidente Frei para manifestar su descontento. Ante la presión de la Casa Blanca, Gabriel Valdés llegó incluso a presentar su renuncia, la que finalmente no fue aceptada por el Jefe de Estado (VALDÉS, 2009, p. 185).

La agitada reacción local e internacional fue la respuesta previsible a la delicada estrategia diplomática de la Cancillería chilena que hemos intentado trazar en estas breves páginas. La administración Frei, esmerada en concebir las relaciones interamericanas en "clave integracionista" (HENRÍQUEZ y VAN KLAVEREN, 2016, p. 346-350), estaba incluso dispuesta a asumir ciertos riesgos para preservar su autonomía en materia de política exterior.

El acuerdo de febrero de 1970 con Cuba -primer paso hacia una relación creciente con La Habana que se consolidaría con la llegada al poder de Salvador Allende- no es más que uno de los tantos testimonios que nos invitan a reexaminar el papel de la Democracia Cristiana chilena en el contexto de Guerra Fría latinoamericana. Estimamos que, tradicionalmente, la originalidad de la diplomacia castrista, objeto recurrente de numerosos análisis, ha eclipsado a otros experimentos, menos espectaculares por cierto, pero que también aspiraron a emanciparse de la tutela de las superpotencias. Como vimos, sin renegar de su pertenencia a la "esfera occidental" (HURTADO, 2016) -tal como la Cuba revolucionaria jamás objetó su identidad socialista-, el gobierno de Frei logró ejercer una política internacional auténtica, transformándose, por momentos, en una fuente de preocupación para las autoridades de la Casa Blanca. Lo que hemos deseado poner en evidencia a lo largo de este artículo, es que el ejemplo de la "Revolución en Libertad" nos brinda una prueba adicional del estatus protagónico del continente latinoamericano, donde no solo se consumieron activamente ideas provenientes del exterior, sino que también surgieron proyectos y doctrinas originales ${ }^{11}$. Esta constatación nos obliga, a su vez, a desechar la imagen de una América Latina compuesta de un conjunto de países sometidos a los imperativos de los "dos grandes" del planeta.

Volviendo al gobierno de Frei, y a modo de epílogo, es importante recalcar que los esfuerzos efectuados en política exterior fueron considerablemente reforzados durante los "mil días" de la Unidad Popular (19701973), ampliando el proyecto de apertura internacional. En efecto, el programa de Allende consagraba un lugar especial a las relaciones con el "mundo socialista", lo que se vio reflejado en el inmediato restablecimiento de los vínculos diplomáticos con la Cuba castrista. Paralelamente, la administración del Presidente Allende optó también por iniciar relaciones oficiales con China y después con la RDA, Corea del Norte y la República Democrática de Vietnam (AMOROS, 2013 , p. 312), pasos que el gobierno anterior no había osado efectuar. No obstante, contrariamente a las pretensiones de Salvador Allende, quien hubiese querido entablar una mayor cooperación con el Este, esta mayor cercanía no condujo a una multiplicación decisiva de la asistencia material. Con el objeto de obtener una ayuda substancial, el Jefe de Estado realizó una ambiciosa gira a la URSS en diciembre de 1972,pero sin obtener los resultados esperados, lo que lo sumió en una profunda decepción (MARTNER, 1988, p. 224-226). En cuanto a la China de Mao, orientada hacia sus propios problemas en tiempos de la Revolución Cultural, un compromiso decidido con

\footnotetext{
${ }^{11}$ Solo por nombrar algunos ejemplos que ilustran la originalidad y el impacto global del pensamiento latinoamericano en los años 1960, podríamos evocar la tesis del "foquismo" articulada por el Che Guevara, la teología de la liberación y la teoría de la dependencia.
} 
la Unidad Popular simplemente no hacía parte de las prioridades, tanto así que Pekín optó por no romper los lazos diplomáticos con Chile después del violento Golpe de Estado liderado por Augusto Pinochet (FRIEDMAN, 2015, p. 169-170, 209) ${ }^{12}$.

Bajo estas circunstancias, lo que a nuestros ojos amerita ser destacado aquí es que muchas características de las relaciones internacionales en tiempos de Allende eran ya perceptibles durante la segunda mitad de la década de 1960. Por consiguiente, debemos entender la ampliación de los vínculos con el "mundo socialista" como un proceso, ciertamente, de indudable intensificación, pero de una intensificación inscrita en una continuidad. Como lo hemos querido demostrar, el gobierno de Eduardo Frei Montalva, evidenciado una osadía inesperada y una resuelta disposición internacional, capaz de irritar a su principal aliado (los Estados Unidos), se posicionó en el continente como un actor relevante, esmerado en defender una agenda propia a riesgo inclusive de debilitar las alianzas tradiciones.

Otros ejemplos reveladores -pensamos en el régimen de Juan Velasco Alvarado en Perú, en la "transformación nacional" de Carlos Lleras Restrepo en Colombia, en el gobierno de Rómulo Betancourt en Venezuela o del General boliviano Juan José Torresmerecerían igualmente un estudio profundo. Todos ellos representan indicios invaluables que debieran conducirnos a recalcar el carácter protagónico de América Latina en la evolución del enfrentamiento global que definió la segunda mitad del siglo $X X$. Enfocarse en el rol de experiencias políticas tradicionalmente poco consideradas, a menudo opacadas ante la espectacularidad del caso cubano, constituye un esfuerzo necesario que nos permitirá, a su vez, conformar una imagen más compleja, más acabada, de las peculiaridades de la Guerra Fría en el continente latinoamericano.

\footnotetext{
${ }^{12}$ El socialista Carlos Altamirano nos ofrece un excelente testimonio de la prudencia de las grandes potencias socialistas hacia el gobierno de Allende. En una de sus giras a la URSS, durante la cual se reunió con Leonid Brézhnev, este último le expresó sin tapujos sus dudas hacia el proyecto de la Unidad Popular. El Secretario General del PCUS expresó "serias dudas de que el socialismo pudiera construirse en democracia", antes de interpelar al visitante con un argumento difícil de contrarrestar: "Mire, considere usted que Chile es el país más distante de la URSS, que no hay otro país que quede tan lejos como el de ustedes". En pocas palabras, Moscú no haría de Chile una segunda Cuba. En cuanto a China, Altamirano recibió una estocada categórica de Zhou Enlai: "Ustedes están en un camino imposible" (POLITZER, 1990, p. 111-113).
}

\section{Referencias}

ALEKSEEV, N. B. Conversación del embajador N. B. Alekseev con VolodiaTeitelboim. Estudios Públicos, Santiago, n. 72, p. 411-413, 1998.

AMORÓS, Mario. Salvador Allende: La biografía. Santiago: Ediciones B, 2013.

Archivo Histórico del Ministerio de Asuntos Exteriores de Chile (AHMAEC). Fondo Países - Rusia. Santiago. Chile.

Archivo Casa Museo Eduardo Frei Montalva (AEF). Fondo 57. Santiago. Chile.

BERNSTEIN, Enrique. Recuerdos de un diplomático. Santiago: Andrés Bello, 1986, v. 2.

BRANDS, Hal. Latin America's Cold War. Cambridge: Harvard University Press, 2010.

CASTRO, Baltazar. Do-a Revolución. Santiago: Nascimento, 1969.

CASTRO, Fidel. Frei y las máscaras del reformismo. Cuba, La Habana, abr. 1966, p. 14-16.

CLISSOLD, Stephen (Org.). Soviet Relations with Latin America, 1918-68: a Documentary Survey. Londres: Oxford University Press, 1970.

DEBRAY, Régis. ¿Revolución en la revolución? La Habana: Casa de las Américas, 1967.

DRAGO, Gonzalo. Vino chileno para Cuba. El Rancagüino, Rancagua, 18 enero 1971, p. 13.

FERMANDOIS, Joaquín. Chile y la "Cuestión Cubana" 19591964. Historia, Santiago, n. 17, p. 113-200, 1982.

FERMANDOIS, Joaquín. Mundo y fin de mundo. Chile en la política mundial 1900-2004. Santiago: Ediciones Universidad Católica de Chile, 2004.

FRIEDMAN, Jeremy. Shadow Cold War: The Sino-Soviet Competition for the Third World. Chapel Hill: University of North Carolina Press, 2015. <https://doi.org/10.5149/northcarolina/ 9781469623764.001.0001>.

GAMBOA, Alberto. Chile romperá el bloqueo: cambia productos con Cuba. Clarin, Santiago, 19 feb. 1970, p. 3.

GONZÁLEZ, Marianne; PALIERAKI, Eugenia (Org.). Revoluciones imaginadas. Itinerario de la idea revolucionaria en América Latina contemporánea. Santiago: RIL Editores, 2013.

GRIGULEVICH, I. R. The Significance of the Christian Democratic Electoral Victory in Chile. In: OSWALD, Gregory (Org.). Soviet Image of Contemporary Latin America: A Documentary History, 1960-1968. Austin: Texas Press, 1970.

HARMER, Tanya. Allende's Chile \& the Inter-American Cold War. Chapel Hill: The University of North Carolina Press, 2011.

HARMER, Tanya; RIQUELME, Alfredo (Org.). Chile y la Guerra Fría global. Santiago: RIL Editores, 2014.

HENRÍQUEZ, María José; VAN KLAVEREN, Alberto. Reformismo y pragmatismo: la política exterior de Eduardo Frei Montalva, 1964-1970. In: HUNEEUS, Carlos; COUSO, Javier (Org). Eduardo Frei Montalva: un gobierno reformista. A 50 a-os de la "Revolución en Libertad". Santiago: Editorial Universitaria, 2016. p. 343-369. 
HURTADO, Sebastián. The Gathering Storm: The United States, Eduardo Frei's Revolution in Liberty and the Polarization of Chilean Politics, 1964-1970. 2016. Tesis (Doctorado) - Ohio University, 2016.

LABARCA, Eduardo. Salvador Allende: Biografía sentimental. Santiago: Catalonia, 2014.

LÉVESQUE, Jacques. L'URSS et la révolution cubaine. París: Presse de la Fondation Nationale des Sciences Politiques, 1976.

MARTNER, Gonzalo. El Gobierno del Presidente Salvador Allende, 1970-1973: una evaluación. Concepción: LAR, 1988.

MEMORIA del Ministerio de Relaciones Exteriores correspondiente al año 1964. Santiago: Editorial Universidad Católica.

MEMORIA del Ministerio de Relaciones Exteriores correspondiente al año 1965. Santiago: Editorial Universidad Católica.

MEMORIA del Ministerio de Relaciones Exteriores correspondiente al año 1968. Santiago: Editorial Universidad Católica.

MORENO, Rafael. Sin Reforma Agraria no habría sido posible. Memorias de la Reforma Agraria Chilena, 1958-1970. Santiago: Ediciones Copygraph, 2014.

PACHECO Máximo; HOLGER, James. Recuerdos de la Unión Soviética. Santiago: Andrés Bello, 2009.

PARTIDO DEMÓCRATA CRISTIANO. Un Programa y un Gobierno. Santiago: Editorial del Pacífico, 1967.
Política Internacional de Chile. Politica y Espíritu, Santiago, n. 288 , p. 6-7, 1965 .

POLITZER, Patricia. Altamirano. Santiago: Melquíades, 1990.

RUPPRECHT, Tobias. Socialist high modernity and global stagnation: a shared history of Brazil and the Soviet Union during the Cold War. Journal of Global History, Cambridge, n. 6, p. 505-528, 2011

RUPPRECHT, Tobias. Soviet Internationalism after Stalin: Interaction and Exchange betweenthe USSR and Latin America during the Cold War. Cambridge: Cambridge University Press, 2015. <https://doi.org/10.1017/CBO9781316212769>.

SPENCER, Daniela; GILBERT, Joseph (Org.). In From the Cold: Latin America's New Encounter with the Cold War. Durham: Duke University Press, 2008.

TURRENT, Isabel. La Unión Soviética en América Latina: el caso de Brasil. Foro Internacional, México, DF, v. 27, n. 1, p. 75-101, 1986.

VALDÉS, Gabriel. Sue-os y memorias. Santiago: Taurus, 2009.

Vino chileno para Cuba: Antología poética. Santiago: Sociedad de Escritores de Chile, 1972.

ZOUREK, Michal. Checoslovaquia y el Cono Sur 1945-1989: Relaciones políticas, económicas y culturales durante la Guerra Fría. Praga: Editorial Karolinum, 2014.

\section{Autor/Author:}

RAFAEL PEDEMONTE rafaelpedemonte@gmail.com

- Historiador y asistente de investigación en la Universidad de Gante, Bélgica, en el marco de un proyecto post-doctoral enfocado en el estudio de los procesos revolucionarios en América Latina durante el siglo XX y, en particular, en la Revolución Cubana. El autor posee el título de Doctor en Historia, otorgado tanto por la Universidad de París 1 Panthéon-Sorbonne como por la Pontificia Universidad Católica de Chile, en un programa de co-tutela. Entre sus principales publicaciones, figuran las siguientes: "'La fin des chasses gardées': l'Alliance atlantique et la 'guerre froide latino-américaine' (1959-1973)", in Relations internationales, v. 4, n. 168, p. 143-160, 2016; "Le sort des lumumbistes chiliens face aux enjeux de la guerre froide (1964-1973)", in Caravelle, n. 108, p. 149-168, 2017; "Una relación tensa y ambivalente: el medio intelectual cubano ante 'lo soviético' en los primeros años revolucionarios (1959-1966)", in Historia, v. 1, n. 50, p. 141-173, 2017; "Birches too difficult to cut down: The rejection an assimilation of the Soviet reference in Cuban culture", in International Journal of Cuban Studies, v. 9, n. 1, p. 127-141, 2017.

- Historiador e trabalha como assistente em seu pós-doutoramento na Universidade de Gante, Bélgica. Atualmente desenvolve projeto dedicado à história da revolução cubana sob uma perspectiva global e o (143iversidade Católica do Chile. Principais publicações: “'La fin des chasses gardées': l'Alliance atlantique et la 'guerre froide latino-américaine' (1959-1973)", in Relations internationales, v. 4, n. 168, p. 143-160, 2016; "Le sort des lumumbistes chiliens face aux enjeux de la guerre froide (1964-1973)", in Caravelle, n. 108, p. 149-168, 2017; "Una relación tensa y ambivalente: el medio intelectual cubano ante "lo soviético' en los primeros años revolucionarios (1959-1966)", in Historia, v. 1, n. 50, p. 141-173, 2017; "Birches too difficult to cut down: The rejection and assimilation of the Soviet reference in Cuban culture", in International Journal of Cuban Studies, v. 9, n. 1, p. $127-141,2017$

- Historian working as a Post-Doctoral Assistant at Ghent University (Belgium). His current research project focuses on the Cuban revolutionary history and on the idea of revolution in the 20th-Century Latin America. He has obtained his Ph.D. from the University of Paris 1 Panthéon-Sorbonne in collaboration with the Catholic University of Chile. He has published a series of articles on the Latin American Cold War, such as: “'La fin des chasses gardées': l'Alliance atlantique et la 'guerre froide latino-américaine' (1959-1973)", in Relations internationales, v. 4, n. 168, p. 143-160, 2016; "Le sort des lumumbistes chiliens face aux enjeux de la guerre froide (1964-1973)", in Caravelle, n. 108, p. 149-168, 2017; "Una relación tensa y ambivalente: el medio intelectual cubano ante 'lo soviético' en los primeros años revolucionarios (1959-1966)", in Historia, v. 1, n. 50, p. 141-173, 2017; "Birches too difficult to cut down: The rejection and assimilation of the Soviet reference in Cuban culture", in International Journal of Cuban Studies, v. 9, n. 1, p. 127-141, 2017. 